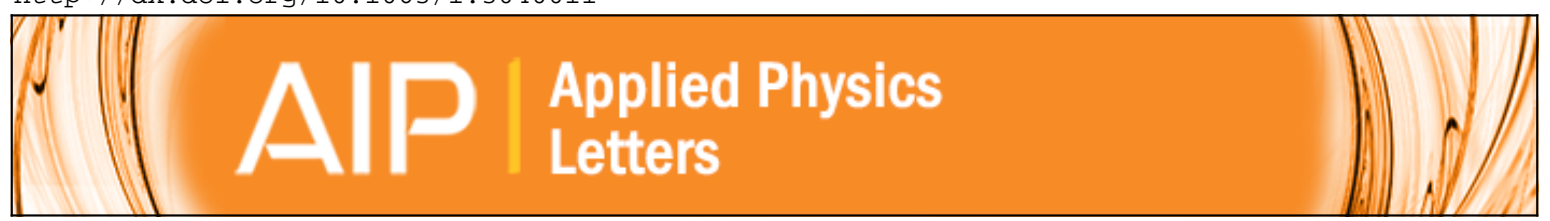

\title{
Power generation and shunt damping performance of a single crystal lead magnesium niobate-lead zirconate titanate unimorph: Analysis and experiment
}

A. Erturk, O. Bilgen, and D. J. Inman

Citation: Applied Physics Letters 93, 224102 (2008); doi: 10.1063/1.3040011

View online: http://dx.doi.org/10.1063/1.3040011

View Table of Contents: http://scitation.aip.org/content/aip/journal/apl/93/22?ver=pdfcov

Published by the AIP Publishing

\section{Articles you may be interested in}

Single-crystal lead magnesium niobate-lead titanate (PMN/PT) as a broadband high power transduction material

J. Acoust. Soc. Am. 121, 2591 (2007); 10.1121/1.2717496

Effect of poling on dielectric anomalies at phase transitions for lead magnesium niobate-lead titanate crystals in the morphotropic phase boundary region

J. Appl. Phys. 99, 064101 (2006); 10.1063/1.2179972

High-field dielectric and piezoelectric performance of soft lead zirconate titanate piezoceramics under combined electromechanical loading

J. Appl. Phys. 96, 6634 (2004); 10.1063/1.1812586

Model of the polarization response of single-crystal lead magnesium niobate-lead titanate for transducer applications

J. Appl. Phys. 94, 5172 (2003); 10.1063/1.1610808

Biased lead zirconate titanate as a high-power transduction material

J. Acoust. Soc. Am. 105, 2248 (1999); 10.1121/1.426830

\section{AlP Re-register for Table of Content Alerts}




\title{
Power generation and shunt damping performance of a single crystal lead magnesium niobate-lead zirconate titanate unimorph: Analysis and experiment
}

\author{
A. Erturk, ${ }^{1, a)}$ O. Bilgen, ${ }^{2}$ and D. J. Inman ${ }^{2}$ \\ ${ }^{1}$ Department of Engineering Science and Mechanics, Center for Intelligent Material Systems and Structures, \\ Virginia Polytechnic Institute and State University, Blacksburg, Virginia 24061, USA \\ ${ }^{2}$ Department of Mechanical Engineering, Center for Intelligent Material Systems and Structures, \\ Virginia Polytechnic Institute and State University, Blacksburg, Virginia 24061, USA
}

(Received 2 July 2008; accepted 8 November 2008; published online 2 December 2008)

\begin{abstract}
This letter investigates the power generation and shunt damping performance of the single crystal piezoelectric ceramic lead magnesium niobate-lead zirconate titanate (PMN-PZT) analytically and experimentally. PMN-PZT is a recently developed interface for energy harvesting and shunt damping with its large piezoelectric constant $(-2252 \mathrm{pm} / \mathrm{V})$ and coupling coefficient $(0.95)$ for the transverse piezoelectric mode. A unimorph PMN-PZT cantilever with an aluminum substrate is tested under base excitation and its electromechanical response is predicted with a coupled distributed parameter model. The power generation performance of the device is $138 \mu \mathrm{W} /\left(g^{2} \mathrm{~cm}^{3}\right)$ at $1744 \mathrm{~Hz}$, causing $84 \%$ tip vibration attenuation due to the resistive shunt damping effect. () 2008 American Institute of Physics. [DOI: 10.1063/1.3040011]
\end{abstract}

There has been an explosion of research in the area of vibration-based energy harvesting over the past decade. ${ }^{1}$ Among the basic transduction mechanisms for vibration-toelectricity conversion (electromagnetic, electrostatic, and piezoelectric transductions), piezoelectric transduction has received the most attention and has been discussed in several review articles. ${ }^{2,3}$ Research on converting ambient vibrations to electricity for powering small electronic components followed the research on piezoelectric shunt damping, ${ }^{4}$ where the main concern was the vibration attenuation in the structure rather than the electrical output. Although the particular interest in the energy harvesting problem is the electrical response and that in the shunt damping problem is the vibration attenuation, in practice, these effects are coupled.

Other than the piezoelectric polymer polyvinylidene fluoride, the two popular piezoelectric materials in the literature of energy harvesting and shunt damping are the soft ceramics PZT-5A and PZT-5H (where PZT stands for lead zirconate titanate). The effectiveness of the energy harvesting process and the shunt damping treatments depends on the electromechanical coupling. The two commonly used piezoelectric modes in energy harvesting and shunt damping are the 31-mode (transverse) and the 33-mode (longitudinal). The bending mode with conventional electrodes uses the 31mode, whereas the stack arrangements as well as the recently introduced active-fiber composites and macrofiber composites (MFCs) utilize the 33-mode. Here, we focus on the commonly employed 31-mode of piezoelectricity for bending vibrations.

The room temperature 31-mode piezoelectric constants $\left(d_{31}\right)$ of PZT-5A and PZT-5H were reported in literature ${ }^{5}$ as -190 and $-265 \mathrm{pm} / \mathrm{V}$, respectively, with the transverse mode coupling coefficients $\left(k_{31}\right)$ of 0.40 and 0.36 , respectively. Recently, the single crystal lead magnesium niobatelead titanate (PMN-PT) was used for designing a high elec-

\footnotetext{
a) Author to whom correspondence should be addressed. Electronic mail: erturk@vt.edu.
}

tromechanical coupling adaptive vibration absorber ${ }^{6}$ in MFC actuators $^{7}$ and also for energy harvesting ${ }^{8}$ due to its unique characteristics. The PMN-PT sample used in the energy harvesting implementation ${ }^{8}$ had a transverse mode piezoelectric constant of $-643 \mathrm{pm} / \mathrm{V}$ with a coupling coefficient of 0.725 . Detailed properties of PMN-PT single crystals can be found in literature, ${ }^{9,10}$ where the transverse mode piezoelectric constant was reported to be as high as $-1330 \mathrm{pm} / \mathrm{V}$ with a coupling coefficient of 0.59 . More recently, PMN-PZT single crystals were grown by the solid state crystal growth method $^{11}$ and their elastic, piezoelectric, and dielectric constants were compared with those of PMN-PT (Refs. 12 and 13) and PZT-5H. ${ }^{12}$ It was reported in Ref. 13 that the transverse piezoelectric constant of PMN-PZT can be as high as $-1440 \mathrm{pm} / \mathrm{V}$ with a coupling coefficient of 0.935 .

In this work, piezoelectric energy harvesting and shunt damping performance of a single crystal PMN-PZT is studied analytically and experimentally. The single crystal PMN-PZT sample $\left(20 \times 5 \times 0.5 \mathrm{~mm}^{3}\right)$ used here has $d_{31}=-2252 \mathrm{pm} / \mathrm{V}$ and $k_{31}=0.95$ according to the manufacturer $^{11}$ (CPSC200-115), which are considerably larger than the previously published values. An aluminum (Al) substrate of $0.79 \mathrm{~mm}$ thickness is bonded to the PMNPZT layer and a PMN-PZT/Al unimorph beam is obtained. Figure 1(a) shows the unimorph with the conductive copper tapes connecting the gold electrodes to the output wires. The unimorph bender is clamped on an electromagnetic shaker as a cantilevered beam with an overhang length of $16 \mathrm{~mm}$ [Fig. 1(b)]. A low mass accelerometer measures the acceleration at the base of the cantilever and a laser vibrometer measures the tip velocity [Fig. 1(c)].

The schematic of the setup is depicted in Fig. 2, where the unimorph cantilever is excited by a harmonic base displacement of amplitude $Y_{0}$ and angular frequency $\omega$. The coupled equations governing the system dynamics are given by ${ }^{14}$ 
(a)

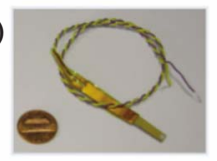

(b)

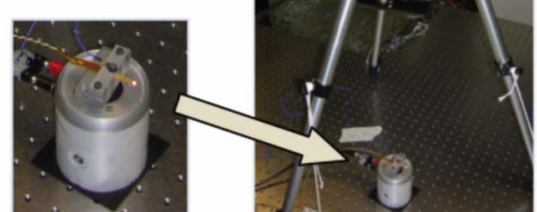

FIG. 1. (Color online) (a) PMN-PZT/Al unimorph analyzed in the experiment, (b) its clamped configuration on the shaker with an accelerometer, and (c) the laser vibrometer measuring the tip velocity.

$$
\begin{gathered}
Y I \widetilde{w}_{x x x x}(x, t)+c_{s} I \widetilde{w}_{x x x x t}(x, t)+c_{a} \widetilde{w}_{t}(x, t)+m \widetilde{w}_{t t}(x, t) \\
+\vartheta v(t)\left[\delta_{x}(x)-\delta_{x}(x-L)\right]=m \omega^{2} Y_{0} e^{j \omega t},
\end{gathered}
$$

and

$$
C_{p} \dot{v}(t)+v(t) / R_{l}=-d_{31} \bar{c}_{11}^{E} h_{\mathrm{pc}} b \int_{0}^{L} \widetilde{w}_{x x t}(x, t) d x,
$$

where $Y I$ is the bending stiffness, $c_{s} I$ is the internal (strain rate) damping term, $c_{a}$ is the external (viscous air) damping term, $m$ is the mass per length, and $\widetilde{w}(x, t)$ is the transverse displacement of the beam relative to its base at point $x$ and time $t$. Furthermore, $\vartheta$ is the piezoelectric coupling term in physical coordinates, $v(t)$ is the voltage across the resistive load $R_{l}, \delta(x)$ is the Dirac delta function, $C_{p}$ is the inherent capacitance of the piezoceramic layer, $d_{31}$ is the piezoelectric constant, $\bar{c}_{11}^{E}$ is the elastic stiffness of the piezoceramic layer at constant electric field with the plane-stress assumption for a beam (i.e., $\bar{c}_{11}^{E}=1 / s_{11}^{E}$, where $s_{11}^{E}$ is its elastic compliance at constant electric field), $b$ is the electrode width, $h_{\mathrm{pc}}$ is the distance of the center of the piezoceramic layer from the neutral axis, and $j$ is the unit imaginary number. Subscripts $x$ and $t$ stand for partial differentiation with respect to space and time, respectively, and an overdot denotes an ordinary differentiation with respect to time.

From the expansion theorem,

$$
\widetilde{w}(x, t)=\sum_{r=1}^{\infty} \phi_{r}(x) \eta_{r}(t)
$$

where $\phi_{r}(x)$ is the mass normalized eigenfunction and $\eta_{r}(t)$ is the modal response of the $r$ th vibration mode.

Using the orthogonality conditions of the eigenfunctions and Eq. (3) in Eq. (1), one obtains ${ }^{14}$

$$
\begin{gathered}
\ddot{\eta}_{r}(t)+2 \zeta_{r} \omega_{r} \dot{\eta}_{r}(t)+\omega_{r}^{2} \eta_{r}(t)+\chi_{r} v(t) \\
=m \omega^{2} Y_{0} e^{j \omega t} \int_{0}^{L} \phi_{r}(x) d x,
\end{gathered}
$$

where $\omega_{r}$ is the undamped natural frequency (for $R_{l} \rightarrow 0$ ), $\zeta_{r}$ is the damping ratio, and $\chi_{r}$ is the modal coupling term of the $r$ th mode in the mechanical equation due to the converse piezoelectric effect.

Substituting Eq. (3) into Eq. (2) yields

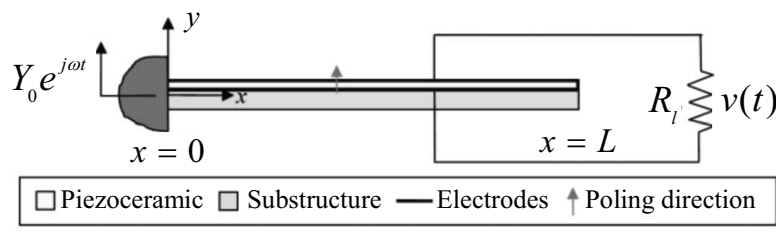

FIG. 2. Unimorph cantilever with a resistive load under base excitation (piezoelectric energy harvester model).

$$
\dot{v}(t)+v(t) / \tau_{c}=\sum_{r=1}^{\infty} \varphi_{r} \dot{\eta}_{r}(t),
$$

where $\tau_{c}=R_{l} C_{p}$ is the time constant of the circuit and $\varphi_{r}$ is the modal coupling term in the circuit equation due to the direct piezoelectric effect.

The steady state solutions of Eqs. (4) and (5) $\operatorname{are}^{14}$

$$
v(t)=\frac{j m \omega^{3} Y_{0} e^{j \omega t} \sum_{r=1}^{\infty} \frac{\varphi_{r} \int_{0}^{L} \phi_{r}(x) d x}{\omega_{r}^{2}-\omega^{2}+j 2 \zeta_{r} \omega_{r} \omega}}{\frac{1+j \omega \tau_{c}}{\tau_{c}}+\sum_{r=1}^{\infty} \frac{j \omega \chi_{r} \varphi_{r}}{\omega_{r}^{2}-\omega^{2}+j 2 \zeta_{r} \omega_{r} \omega}}
$$

and

$$
\widetilde{w}(x, t)=\sum_{r=1}^{\infty} \frac{\left[m \omega^{2} Y_{0} e^{j \omega t} \int_{0}^{L} \phi_{r}(x) d x-\chi_{r} v(t)\right] \phi_{r}(x)}{\omega_{r}^{2}-\omega^{2}+j 2 \zeta_{r} \omega_{r} \omega},
$$

which can be used to extract the voltage output-to-base acceleration and the tip displacement-to-base acceleration frequency response functions (FRFs).

The overhang dimensions of the unimorph cantilever [Fig. 1(b)] are $16 \times 5 \times 1.33 \mathrm{~mm}^{3}$ due to an epoxy layer of $\sim 0.04 \mathrm{~mm}$ thickness. In the experiments, 14 different resistive loads (from $10 \Omega$ to $685 \mathrm{k} \Omega$ ) are used. Because PMNPZT is very brittle, the cantilever is not clamped very tightly, resulting in the experiment having a more flexible boundary than the theory. The bending stiffness $Y I$ is estimated by using the experimental first natural frequency $(1744 \mathrm{~Hz}$ for $10 \Omega$ load resistance) and the theoretical mass per length $(m=0.0304 \mathrm{~kg} / \mathrm{m})$ as $Y I=0.0194 \mathrm{~N} \mathrm{~m}^{2}$. The capacitance of the original $20 \mathrm{~mm}$ long sample was reported by the manufacturer ${ }^{11}$ as $8.88 \mathrm{nF}$. Thus, for an overhang length of $16 \mathrm{~mm}, C_{p}=7.10 \mathrm{nF}$ is used here. Note that the reported ${ }^{11}$ elastic compliance of PMN-PZT is as large as $s_{11}^{E}$ $=127 \mathrm{pm}^{2} / \mathrm{N}$.

For 14 different resistive loads, the experimentally measured voltage FRFs (per base acceleration in $g$ - gravitational acceleration) are shown in Fig. 3(a), whereas the theoretical (a) Experimental

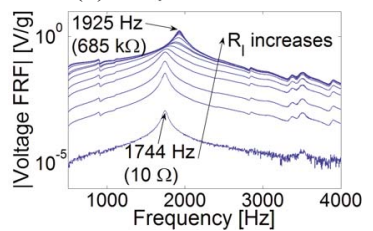

(b) Analytica

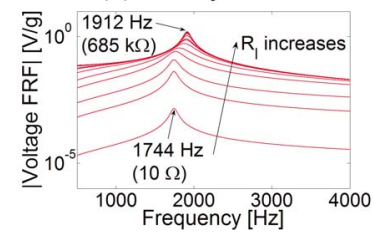

FIG. 3. (Color online) Voltage output-to-base acceleration FRFs for 14 different resistive loads: (a) experimental and (b) analytical. 

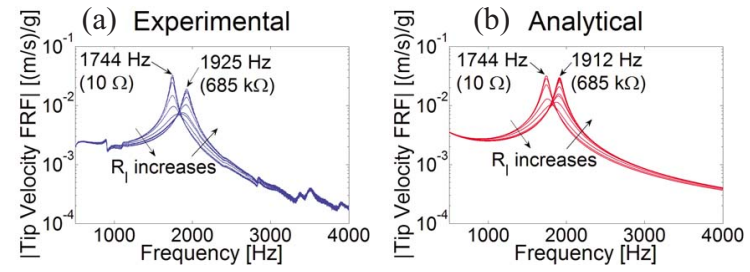

FIG. 4. (Color online) Tip velocity-to-base acceleration FRFs for 14 different resistive loads: (a) experimental and (b) analytical.

predictions of Eq. (6) are displayed in Fig. 3(b). Note that as the load resistance is increased from $10 \Omega$ to $685 \mathrm{k} \Omega$, the first resonance frequency moves from the short circuit resonance frequency $(1744 \mathrm{~Hz})$ to the open circuit resonance frequency (which is $1925 \mathrm{~Hz}$ in the experimental FRF and $1912 \mathrm{~Hz}$ in the analytical FRF). The analytical model has a $0.7 \%$ error in predicting the open circuit resonance frequency of the first mode. The large frequency shift with changing load resistance is a result of large piezoelectric coupling.

Equation (7) can be modified to give the absolute velocity response at the tip of the cantilever in order to predict the laser vibrometer measurement. Then, the tip velocity measurements shown in Fig. 4(a) can be predicted as in Fig. 4(b). In all FRFs shown in Figs. 3 and 4, the mechanical damping ratio is $2.2 \%$ (identified once for $10 \Omega$ load resistance). Due to the shift in the resonance frequency, the vibration amplitude is attenuated for excitations around $1744 \mathrm{~Hz}$, whereas it is amplified around $1925 \mathrm{~Hz}$.

For excitation at $1744 \mathrm{~Hz}$, variation in the electrical power with load resistance is given by Fig. 5(a). The maximum power of $14.7 \mu \mathrm{W} / g^{2}$ is obtained for an optimum resistive load of $2.7 \mathrm{k} \Omega$. The overhang volume and the mass of the cantilever are about $0.106 \mathrm{~cm}^{3}$ and $0.486 \mathrm{~g}$, respectively. The maximum power density (power per volume) and the specific power (power per mass) of the device are then $138 \mu \mathrm{W} /\left(g^{2} \mathrm{~cm}^{3}\right)$ and $30.2 \mathrm{~mW} /\left(g^{2} \mathrm{~kg}\right)$. Due to the resistive shunt damping effect associated with the electrical power generation, the vibration amplitude at the tip of the
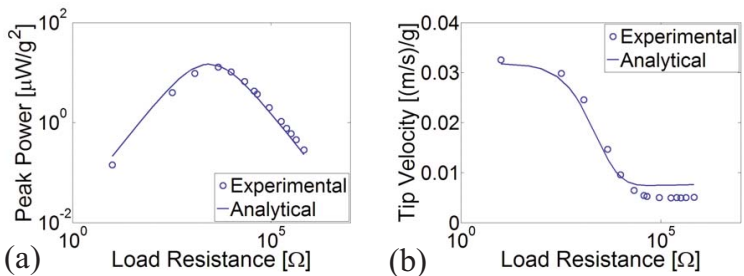

FIG. 5. (Color online) Variations in the (a) electrical power and the (b) tip velocity amplitudes with load resistance for excitation at $1744 \mathrm{~Hz}$.

cantilever is reduced by $84 \%$ according to Fig. 5(b). The model predictions are very good in Figs. 3-5. PMN-PZT is a good interface for energy harvesting and especially for shunt damping due to its large piezoelectric coupling.

The authors gratefully acknowledge the support of the AFOSR MURI under Grant No. F 9550-06-1-0326 monitored by Dr. B. L. Lee and the Ceracomp Corporation for providing the single crystal ceramics.

${ }^{1}$ S. P. Beeby, M. J. Tudor, and N. M. White, Meas. Sci. Technol. 17, R175 (2006).

${ }^{2}$ S. R. Anton and H. A. Sodano, Smart Mater. Struct. 16, R1 (2007).

${ }^{3}$ S. Priya, J. Electroceram. 19, 167 (2007).

${ }^{4}$ G. A. Lesieutre, Shock Vib. Dig. 30, 187 (1998).

${ }^{5}$ M. W. Hooker, NASA Report No. NASA/CR-1998-208708, 1998.

${ }^{6}$ R. Rusovici, J. J. Dosch, and G. A. Lesieutre, J. Intell. Mater. Syst. Struct. 13, 705 (2002).

${ }^{7}$ W. K. Wilkie, D. J. Inman, J. M. Lloyd, and J. W. High, J. Intell. Mater. Syst. Struct. 17, 15 (2006).

${ }^{8}$ A. Badel, A. Benayad, E. Lefeuvre, L. Lebrun, C. Richard, and D. Guyomar, IEEE Trans. Ultrason. Ferroelectr. Freq. Control 53, 673 (2006).

${ }^{9}$ R. Zhang, B. Jiang, and W. Cao, J. Appl. Phys. 90, 3471 (2001).

${ }^{10}$ H. Cao, V. H. Schmidt, R. Zhang, W. Cao, and H. Luo, J. Appl. Phys. 96, 549 (2004).

${ }^{11}$ Ceracomp Co., Ltd. (www.ceracomp.com).

${ }^{12}$ S. Zhang, S. M. Lee, D. H. Kim, H. Y. Lee, and T. R. Shrout, Appl. Phys. Lett. 90, 232911 (2007).

${ }^{13}$ S. Zhang, S. M. Lee, D. H. Kim, H. Y. Lee, and T. R. Shrout, J. Appl. Phys. 102, 114103 (2007).

${ }^{14}$ A. Erturk and D. J. Inman, ASME J. Vibr. Acoust. 130, 041002 (2008). 